\title{
Natal teeth: a sign of fortuity or grave misfortune
}

\author{
R. Sothinathan ${ }^{1}$ and K. Shakib ${ }^{2}$
}

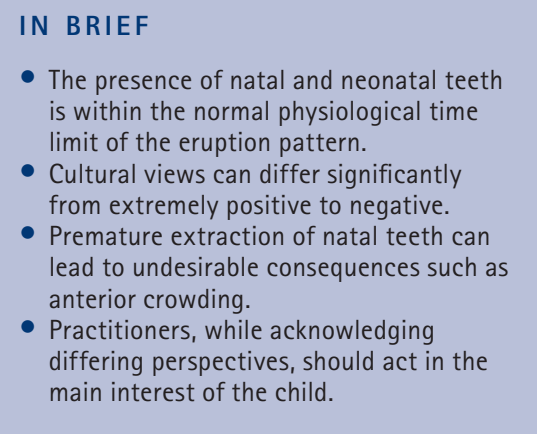

Natal teeth are defined as those present in the mouth at birth. They are uncommon and most frequently occur in the anterior mandible as a pair of primary incisors. They are usually small and poorly developed with little or no root formation. Only 10\% of these teeth are supernumerary. Problems arising are difficulty suckling, severe ulceration of the child's tongue or the mother's breast and the risk of aspiration. These situations would warrant extraction. If the natal teeth are firm and asymptomatic, conservative management is indicated. Negative cultural attitudes towards natal teeth demand good parental counselling and vigilant management in relation to child protection.

\section{INTRODUCTION}

The eruption of a baby's first tooth typically occurs around the age of six months. ${ }^{1}$ It represents an important milestone in the child's first year of dental development. Aberrant eruption patterns can cause anxiety for the child's family. This review examines the historical, aetiological and clinical management aspects of natal teeth. Psycho-sociological and child protection issues associated with natal teeth are also highlighted.

\section{REVIEW}

Teeth present at birth are termed natal teeth, whereas teeth that erupt within the first month of life are termed neonatal teeth. ${ }^{2}$ Natal teeth are also known as congenital teeth, fetal teeth, dentitia praecox and predeciduous teeth. ${ }^{3}$

Babies born with teeth have been recorded since the Roman and Ancient Greek eras and the phenomenon is steeped in superstition, folklore and hearsay.

\footnotetext{
Senior House Officer, Oral \& Maxillofacial Surgery Unit, The Royal London Hospital, First Floor, John Harrison House, Philpot Street, Whitechapel, London, E1 1BB: ${ }^{2 *}$ Consultant in Oral \& Maxillofacial Surgery, Department of Maxillofacial Surgery, Northwick Park Hospital, Watford Road, Middlesex, HA1 3UJ

${ }^{*}$ Correspondence to: Mr Kaveh Shakib

Email:k.shakib@btconnect.com
}

\section{Refereed Paper}

Accepted 3 November 2010

DOI: 10.1038/sj.bdj.2011.200

${ }^{\circledR}$ British Dental Journal 2011; 210: 265-266
Cuneiform inscriptions at Nineveh confirm knowledge of natal teeth in ancient times. ${ }^{4}$ Misconceptions surrounding natal and neonatal teeth vary from beliefs of them being very positive, heralding exceptional favour, and at the other extreme, evil and bearing grave misfortune. ${ }^{3,5}$ In Europe, it was believed that a splendid future awaited such people. ${ }^{6}$ There are descriptions of Ural-Altaic tribes stating that children born with teeth would become witches and sorcerers. ${ }^{6}$ In parts of Africa, affected children have been reported to have been left to die in the bush..$^{5,7}$ In China these teeth are considered bad luck and Allwright reports the parents of one newborn making strong claims for extraction and deposition of the tooth and its evil spirits in the bottom of Hong Kong Harbour. ${ }^{5}$

Lower primary incisors present at birth are common in many mammals ${ }^{6}$ but the incidence in humans varies from 1:800 to 1:6,000 live births. ${ }^{1,8}$ Females have a greater predilection. Natal teeth are three times more common than neonatal teeth. ${ }^{9}$

There is a strong suggestion of familial trait and a positive history has been demonstrated in $8-62 \%$ of cases. ${ }^{10}$ Natal teeth have been associated with some syndromes including chondroectodermal dyplasia, craniofacial dysostosis and Sotos syndrome. ${ }^{1,7}$

Natal teeth are most frequently seen in the anterior mandible (85\%), followed by the anterior maxilla (11\%) and the mandibular premolar/molar region (3\%), usually in pairs. ${ }^{10}$ The strong relation to the mandibular incisor region could be attributed to the normal expected eruption order of the primary dentition. The majority of natal teeth are components of the normal primary dentition. Less than 10\% have been reported as supernumerary. ${ }^{11}$

The exact aetiology of natal teeth remains elusive. Possible theories include genetic predilection. Limrick in 1893 reported natal teeth in a mother, her son and her sister's daughter. ${ }^{12}$ Bodenhoff and Gorlin demonstrated that 15\% of babies with natal teeth had close relatives who claim to have presented with a similar scenario. ${ }^{3}$ Hereditary influences are also seen in Massler and Savara's study in ten out of 24 cases. $^{2}$ Others suggest endocrinological disturbances, maternal nutritional deficiency or malaise during gestation as the cause. ${ }^{8}$ It has been claimed that early osteoblastic activity in the area of the tooth germ and environmental factors increase the incidence of natal teeth. ${ }^{7,9}$ The generally accepted theory is that an anterior, superficial position of the tooth germ due to underlying hereditary influences is

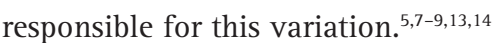

Clinically, natal teeth are small, conical and usually have an immature appearance with poorly-developed, hypoplastic enamel and dentine. ${ }^{7,8}$ They are attached to the mucosa and are occasionally covered by a pad of soft tissue. There is usually absent 
or poor root development and this generally results in significant mobility. ${ }^{5,7,8,10}$

The diagnosis of natal teeth must follow a thorough clinical examination of the patient under ample lighting. The maxillofacial surgery team is the usual point of referral in most cases.

Natal teeth can be classified in four categories: oedema of the gingival tissue with an unerupted but palpable tooth, eruption of the incisal margin of the crown, a solid crown attached loosely to the alveolus with little or no root or a shell-like crown loosely attached with little or no root., ${ }^{4,7}$ Differential diagnoses include Bohn's nodules, gingival epulis or hamartomas of the alveolus. ${ }^{7}$

Management of natal teeth is typically conservative. Clinical indications for removal include:

- Poor support and ensuing mobility as there is a significant aspiration risk $^{3,5,7-9,14}$

- Inability to latch and feed $3,5,7-9,14$

- Interference with suckling 3,5,7-9,14

- Breast irritation detrimental to feeding $^{3,5,7-9,14}$

- Persistent lingual ulceration (RigaFede disease) that is not amenable to conservative management ${ }^{3,5,7-9,14}$

- Supernumerary natal teeth as confirmed by radiography. ${ }^{11}$

Mobile teeth not posing a significant risk of aspiration should be kept under review. Normal root development improves the stability. Kates et al. noted that natal teeth that survived to four months had good prognosis for retention. ${ }^{11}$

Removal is not indicated if there is no interference with feeding. Riga-Fede disease is not an absolute indication for extraction. ${ }^{15}$ The initial treatment for lingual ulceration is either the smoothing of the sharp incisal edges or the placement of adhesive restoration to round off the edges. However, most natal teeth show significant evidence of enamel hypomineralisation and hypoplasia, posing problems with bonding and the risk of aspiration of the material. ${ }^{15}$

The consequence of early extraction of natal teeth is the possibility of anterior crowding, since the space originally occupied by the extracted teeth may be encroached upon by neighbouring primary teeth.

It is important to consider natal teeth as a variation of normal dental development. Aside from the clinical guidelines, healthcare professionals should acknowledge social and cultural attitudes towards natal teeth.

It is quite possible to encounter parents from cultures and communities where this variation is seen as an omen of bad fortune and evil., ${ }^{3,5,6}$ The authors have experienced parents demanding extractions without acceptable indications and it is important for practitioners to acknowledge historical perspectives in reassuring parents and protecting children. Anecdotal reports of fathers' removing neonates' teeth at home raise complex child protection issues. Where there isn't an indication to extract natal teeth, doctors are faced with the difficult decision to balance the best interests of the child and its protection against cultural sensitivity. Compounding this is the debate about acceptability and what is seen as normal practice both in the eyes of the law and socially: for example, male circumcision is largely accepted on cultural grounds alone without any clear clinical indications. Does the refusal to remove natal teeth on the whim of parents fall into a similar trap? Most likely not, but it is important to acknowledge the socio-psychological factors at play when addressing such issues.

In conclusion, neonates with prematurely erupted teeth must be managed with careful clinical examination and treatment planning. It is also important to include parental counselling and awareness as part of the management of natal teeth in order to allay fears and banish myths that may be held. Further research into aetiology would be helpful in this regard and clinicians must be aware of the child protection element that this relatively infrequent occurrence may entail.

1. Uzamis $\mathrm{M}, \mathrm{Olmez} \mathrm{S}, \mathrm{Ozturk} \mathrm{H}, \mathrm{Celik} \mathrm{H}$. Clinical and ultrastructural study of natal and neonatal teeth. J Clin Pediatr Dent 1999; 23: 173-177.

2. Massler M, Savara B S. Natal and neonatal teeth. J Pediatr 1950; 36: 349-359.

3. Bodenhoff J, Gorlin R J. Natal and neonatal teeth: folklore and fact. Pediatrics 1963; 32: 1087-1093.

4. To E W. A study of natal teeth in Hong Kong Chinese. Int J Paediatr Dent 1992; 1: 73-76.

5. Allwright W C. Natal and neonatal teeth: a study among Chinese in Hong Kong. Br Dent J 1958; 105: 163-172

6. Gardiner J H. Erupted teeth in the newborn. Proc R Soc Med 1961; 54: 504-506.

7. Leung A K. Natal teeth. Am J Dis Child 1986; 140: 249-251.

8. Gonçalves F A, Birmani E G, Sugayai N N, Melo A M. Natal teeth: Review of literature and report of an unusual case. Braz Dent J 1998: 9: 53-56.

9. Rao R S, Mathad S V. Natal teeth: case report and review of literature. J Oral Maxillofac Pathol 2009; 13: 41-46.

10. Zhu J, King D. Natal and neonatal teeth. ASDC J Dent Child 1995; 62: 123-128.

11. Kates $G$ A, Needleman $H$ L, Holmes L B. Natal and neonatal teeth: a clinical study. J Am Dent Assoc 1984; 109: 441-443.

12. Limrick O E B. Born with teeth. Lancet 1893; 2: 965

13. Masatomi Y, Abe K, Ooshima T. Unusual multiple natal teeth: case report. Pediatr Dent 1991; 13: $170-172$.

14. Portela M B, Damasceno L, Primo L G. Unusual case of multiple natal teeth. J Clin Pediatr Dent 2004; 29: 37-39.

15. Goho C. Neonatal sublingual traumatic ulceration (Riga-Fede disease): reports of cases. ASDC J Dent Child 1996; 63: 362-364. 